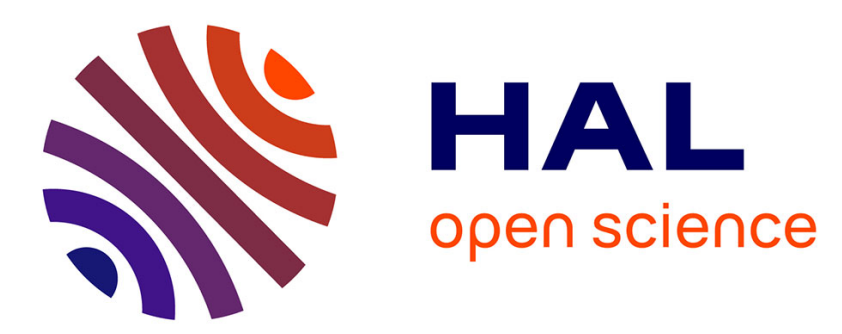

\title{
Twin-image noise reduction by phase retrieval in in-line digital holography
}

Loïc Denis, Corinne Fournier, Thierry Fournel, Christophe Ducottet

\section{To cite this version:}

Loïc Denis, Corinne Fournier, Thierry Fournel, Christophe Ducottet. Twin-image noise reduction by phase retrieval in in-line digital holography. Wavelets XI, SPIE's Symposium on Optical Science and Technology, Aug 2005, San Diego, United States. pp.59140J, 10.1117/12.617405 • ujm-00116996

\section{HAL Id: ujm-00116996 https://hal-ujm.archives-ouvertes.fr/ujm-00116996}

Submitted on 3 Jun 2008

HAL is a multi-disciplinary open access archive for the deposit and dissemination of scientific research documents, whether they are published or not. The documents may come from teaching and research institutions in France or abroad, or from public or private research centers.
L'archive ouverte pluridisciplinaire HAL, est destinée au dépôt et à la diffusion de documents scientifiques de niveau recherche, publiés ou non, émanant des établissements d'enseignement et de recherche français ou étrangers, des laboratoires publics ou privés. 


\title{
Twin-image noise reduction by phase retrieval in in-line digital holography
}

\author{
Loïc Denis, Corinne Fournier, Thierry Fournel, Christophe Ducottet \\ Laboratoire Traitement du Signal et Instrumentation, UMR CNRS 5516, Bâtiment F, \\ 10 rue Barrouin, 42000 Saint-Etienne, France
}

\begin{abstract}
In-line digital holography conciles the applicative interest of a simple optical set-up with the speed, low cost and potential of digital reconstruction.

We address the twin-image problem that arises in holography due to the lack of phase information in intensity measurements. This problem is of great importance in in-line holography where spatial elimination of the twinimage cannot be carried out as in off-axis holography. Applications in digital holography of particle fields greatly depend on its suppression to reach greater particle concentrations, keeping a sufficient signal to noise ratio in reconstructed images. We describe in this paper methods to improve numerically the reconstructed images by twin-image reduction.
\end{abstract}

Keywords: Digital holography, twin-image, phase retrieval, continuous wavelets

\section{INTRODUCTION}

Since the development of high resolution cameras, digital holography is applied to many different fields such as microscopy, particle image velocimetry and deformation analysis. The numerical reconstruction offers a wide range of possibilities for information recovery over the traditional optical reconstruction. The hologram recording as well as the optical reconstruction can be described with the Fresnel Transform. The phase retrieval problem in in-line holography has already been described in litterature. ${ }^{1-8}$ We describe and extend to three dimensional objects both iterative and non-iterative approaches. A new iterative method inspired from non-linear filtering operations based on continuous wavelet transform is introduced. This approach does not require a second hologram.

Let us recall the mathematical framework used in digital holography.

\section{Modeling the diffraction}

The complex amplitude expression of a plane wave after the crossing of a given (complex) transmittance plane $\underline{t}(x, y)$ is given by the Rayleigh-Sommerfeld diffraction formula, valid within the hypotheses of scalar diffraction theory ${ }^{9}$. Using Fresnel paraxial approximation, the reconstructed complex amplitude at distance $z$ from the hologram can be connected with the transmittance function through Fresnel function ${ }^{10}$ $\underline{h_{z}}=1 /(j \lambda z) \exp \left(j \pi\left(x^{2}+y^{2}\right) /(\lambda z)\right)$ (with $j$ the unitary imaginary number, $\lambda$ the wavelength and $x$ and $y$ the spatial variables):

$$
\underline{A_{z}}(x, y)=\underline{t}(x, y) * \underline{h_{z}}(x, y),
$$

where $*$ stands for the two-dimensional spatial convolution operator.

The complex amplitude $\underline{a_{z}}$ is called the Fresnel Transform of transmittance function $\underline{t}$ with parameter $\lambda z$ (scale parameter).

Fresnel Transform has many interesting properties ${ }^{11}$. The most fundamental one is duality property:

$$
\underline{h_{z}} * \underline{h}^{*}=\underline{h_{z}} * \underline{h_{-z}}=\delta
$$

Further author information: (Send correspondence to Loïc Denis)

Loïc Denis: E-mail: loic.denis@univ-st-etienne.fr 


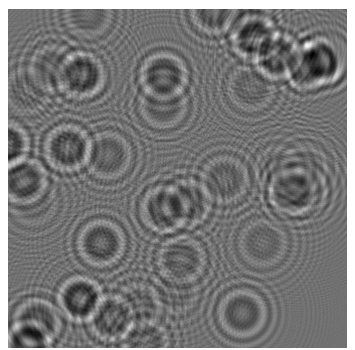

Figure 1. Hologram of opaque spheres computer generated as described in the text

which can be seen as an expression of the principle of ray reversibility ( $\delta$ denotes Dirac's distribution).

Another usefull property is that of additivity:

$$
\underline{h_{z_{1}}} * \underline{h_{z_{2}}}=\underline{h_{z_{1}+z_{2}}}
$$

directly connected with wave propagation.

Fresnel transform is a totally redundant scaling transform. ${ }^{12}$ It does not fulfill wavelets admissibility conditions. A gaussian windowing can be applied to the kernel to limit both its spatial and frequency supports and therefore verify the location condition ${ }^{13}$. The introduction of such a function however appears poorly adapted to the high frequency content of holograms.

Nonredundant multiresolution analysis well-suited for Fresnel holograms were introduced in Ref. 11 in order to perform discrete algorithms dedicated to their reconstruction and image processing (filtering, focusing). ${ }^{14}$ For reconstructing such an hologram from a sum of narrow Gaussian-like functions at a fine scale, the equivalent basis functions on the hologram have to be optimal in the sense of the uncertainty relation. ${ }^{11}$ These last functions, the Frenelets, are the Fresnel transform of B-spline wavelets which asymptotically converge to Gabor functions. ${ }^{15}$

A particular class of three-dimensional objects of applicative interest in fluid mechanics leads to a simple expression of the complex amplitude in the hologram plane: the case of opaque spheres spread in a volume. Under some conditions of low concentration the hologram can be described as the sum of the Fresnel Transforms of the particle apertures. ${ }^{13}$ Figure 1 shows a simulated hologram of particles based on this sum.

\section{Reconstructing the diffractive object}

In optical holography, the information contained in the hologram is recovered after wet chemical processing of the holographic plate by illuminating the revealed hologram with the same light source as used for the recording step. This reconstruction which consists in the diffraction of an incident plane wave on the hologram can be numerically mimicked by taking the intensity of Fresnel Transform at different scales of the hologram.

Pan and Meng ${ }^{16}$ underlined that the use of part of the complex amplitude available via the digital reconstruction facilitated the objects location. It is possible to go further into the numerical treatments of the hologram to recover the information of the object(s).

\section{THE “TWIN-IMAGE" PROBLEM IN IN-LINE HOLOGRAPHY}

\subsection{Experimental setups}

The in-line holography recording setup, also named "Gabor holography" after its inventor, involves no imaging lens. The intensity of the incident coherent beam, diffracted when crossing the object and free propagated beyond is recorded on the sensor (a digital camera) (see figure 2). The reference wave and the object wave are not separate beams but a unique one, this gives a good stability to the setup in adequation with industrial application constraints. The drawbacks of this experimental setup however is the restriction to small opaque or 


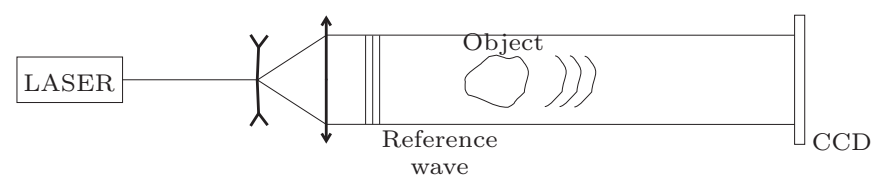

Figure 2. In-line digital holography setup

bigger but transparent objects. The in-line geometry leads to a superposition of both the real and virtual images in the reconstruction step.

Since off-axis geometry description, ${ }^{17}$ this two beams setup has been widely used in optical wavelengths holography. This setup allows for spatial separation of the real and virtual (i.e. twin) images.

Other setups such as phase-shifting holography ${ }^{18}$ were proposed to get rid of the twin image.

Although in-line holography does not record the phase, it is still used today for two major reasons. The first one is that it is hardly possible to realize the off-axis setup for short wavelengths such as that used in soft X-ray holography. ${ }^{1} \quad$ The second reason is the higher resolution available for the study of small objects as in digital holography particle image velocimetry (DHPIV, see Ref. 19 for example). Therefore for such applications, a numerical suppression of the twin-image is wished.

\subsection{Hologram recording: origin of the twin image}

In practice, the complex amplitude of the incident field cannot be directly recorded and only its squared modulus is stored in the sensor: $I_{z}=\left|\underline{A_{z}}\right|^{2}$. If we introduce the opacity function of the object $\underline{a}=1-\underline{t}$, the intensity expression can be expanded using equation 1 as:

$$
I=1-\underline{a} * \underline{h}_{z}-\underline{a}^{*} * \underline{h}_{z}^{*}+\left|\underline{a} * \underline{h_{z}}\right|^{2} .
$$

For the recorded hologram to be workable the last (non linear) term has to be negligible compared with the others. This is a classical condition in in-line holography required for good quality reconstructions. Translucent and low concentration clouds of small opaque objects fulfill this condition. For this class of objects equation 4 can be rewritten as:

$$
\tilde{I} \approx-\underline{a} * \underline{h_{z}}-\underline{a}^{*} * \underline{h_{z}}{ }^{*}
$$

with $\tilde{I}$ denoting the centered image (the hologram minus its mean). The measured intensity in this last expression can be physically interpreted as the recording of both the object diffracting in the positive direction and its symmetric (i.e. conjuguate) diffracting towards the opposite direction. The reconstruction process described in section 1 applied to the measured intensity therefore leads to the superposition of the in-focus image and the out-of-focus twin image: $\tilde{I} * h_{-z} \approx-\underline{a}-\underline{a}^{*} * h_{-2 z}$ The lack of phase information in the hologram plane therefore produces a twin image in either optical or numerical reconstructions.

\subsection{Twin image characteristics}

Figure 3 illustrates the twin-image phenomenon. Small diffracting objects where used to simulate the complex amplitude in a recording plane. The intensity of the field has been stored and two reconstructions have been performed. Image (a) has been computed from the simulated complex amplitude. It is therefore free of the twin-image phenomenon. Only two of the three objects are in focus. The third one is slightly out-of-focus. Image (b) is the result of the simulated hologram reconstruction. The lack of phase information results in the presence of the twin-image. Low frequency fringes appear in image (b) that could not be seen in image (a): this is the so-called "twin-image".

Let us describe the amplitude and spatial localization of the twin image and their dependance with the recording distance $z$. The in-focus image amplitude to out-of-focus twin image amplitude ratio is proportional to the inverse of the recording distance $z$. The twin image is centered on the real image and its value increases radially from zero to its maximum at $r=\sqrt{\lambda z}$ (i.e. $r$ verifying $\pi r^{2} / 2 \lambda z=\pi / 2$ ). At small recording distances, the contrast between the image and its twin is low and both images merge. 


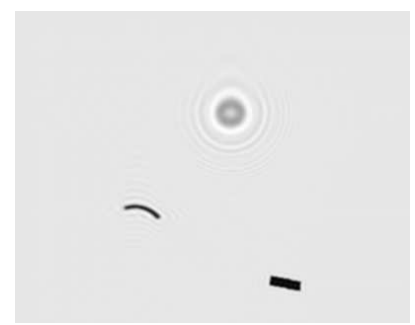

(a)

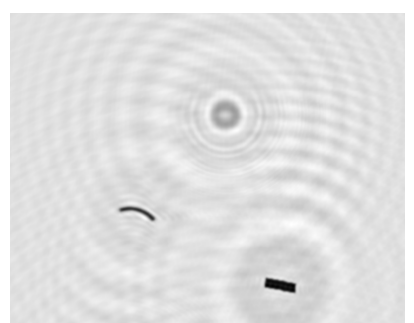

(b)

Figure 3. Twin-image appearing at the reconstruction step: (a) "twin-image free" reconstruction using the phase; (b) classical reconstruction from the hologram, showing the twin-image

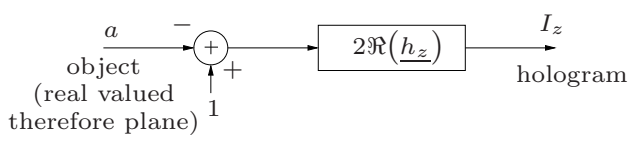

(a)

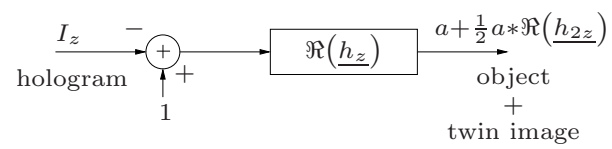

(b)

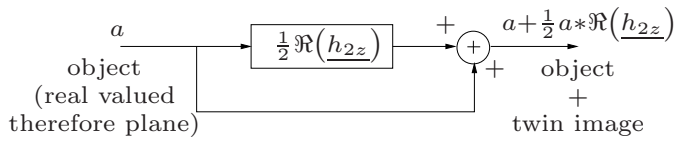

(c)

Figure 4. 2D linear system representations of: (a) the recording step ${ }^{2}$, (b) the reconstruction step by use of real part of complex amplitude, (c) the complete system: recording + numerical reconstruction

When reconstructing a hologram of a large collection of small objects (such as particle assemblies), the twinimage becomes a superimposition of many interference fringe systems. The resulting interference pattern is a speckle whose grain size is to be confused with in-focus object images. ${ }^{20}$

\section{TWIN-IMAGE NOISE REDUCTION TECHNIQUES BASED ON A PAIR OF HOLOGRAMS}

The twin-image noise reduction problem can be formulated as a phase-retrieval problem in the hologram's plane. Both problems are equivalent (see section 2.2). We will write and compare the two classes of approaches (deconvolution or iterative) that have been addressed to the twin-image problem in a "phase-retrieval" formulation. Both approaches require to record a pair of holograms of the same object at different values of the product $\lambda z$. The holograms can either be located at different distances or recorded at different wavelengths.

\subsection{Linear approach: inverse filtering}

The twin-image can be filtered using a physical approach equivalent to deconvolution.

\subsubsection{Formulating the twin-image suppression as a deconvolution problem}

Onural and Scott have reinterpreted the recording and reconstruction steps classically used in digital inline holography in terms of linear systems ${ }^{2}$. The recording step in presence of mostly transparent real objects can be linearly approximated (figure 4(a)). The digital reconstruction step as described in section 1 has the same impulse response (figure 4(b)) (see Ref. 2). The global system therefore is equivalent to that given in figure 4(c) leading to the superposition of the object and its twin image. 


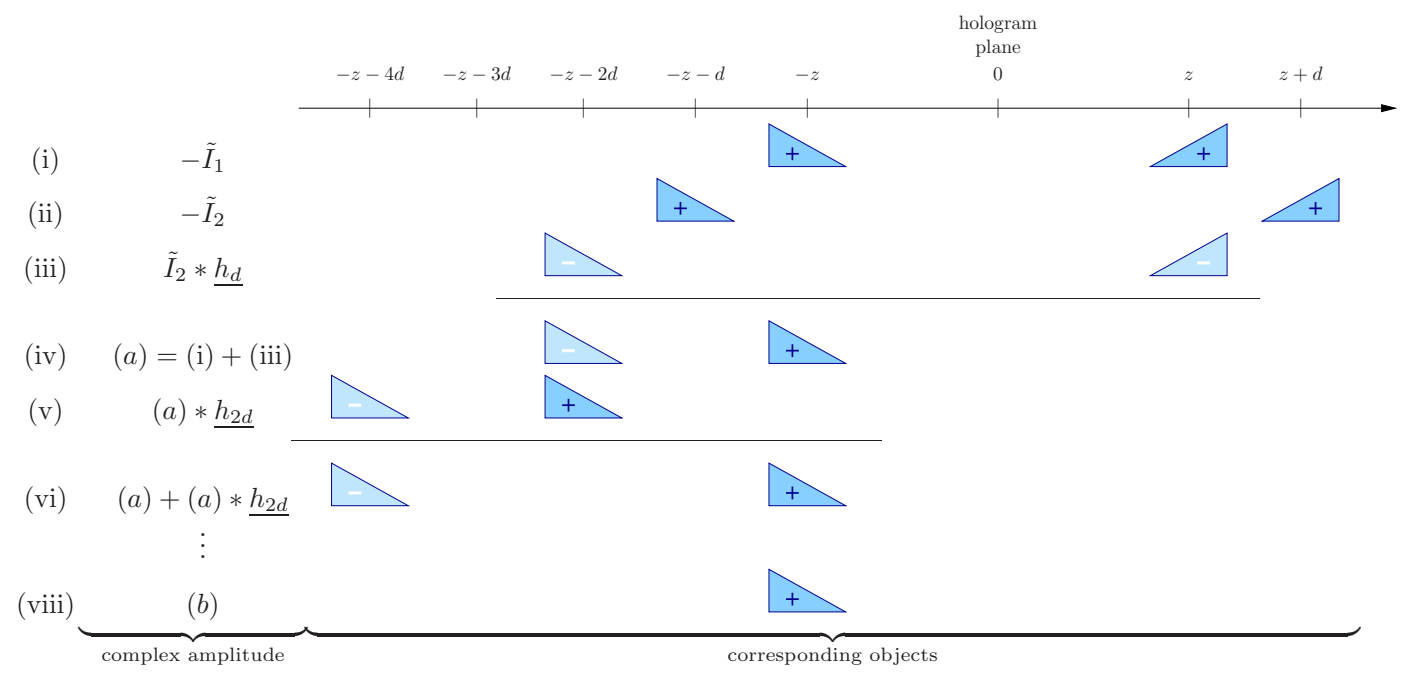

Figure 5. Physical interpretation of the deconvolution filter

Onural and Scott then proposed a new reconstruction system chosen in order to inverse the recording system. The exact inverse does not exist as the transfer function associated with the recording step has many zeros. Pseudo-inverses however can be searched. The one introduced by Onural and Scott ${ }^{2}$ has an interesting physical interpretation.

The strength of digital holography compared to other imaging techniques lies in the three dimensional information recording capability. Although the inverse filtering approach has been applied to plane objects ${ }^{2-4}$ (real or complex valued), the extension to volume objects will have a practical importance. As soon as we study objects that are either volumic or complex valued, two holograms are necessary to suppress the twin-image in an inverse filtering approach. Contrary to previous work, we suggest to express the deconvolution problem as a phase retrieval problem. The searched complex amplitude therefore is that in the hologram plane.

Let us formulate here the deconvolution problem from the two holograms $I_{1}$ and $I_{2}$ recorded respectively at distances $z$ and $z+d$, using equation 5 and properties 2 and 3:

$$
\underbrace{-\tilde{I}_{1}+\tilde{I}_{2} * \underline{h_{d}}}_{\text {(a) }}=\underbrace{a * \underline{h_{z}}}_{\text {(b) }} * \underbrace{\left(\delta-\underline{\left.h_{2 d}\right)}\right.}_{\text {(c) }} .
$$

Term (a) is computed from the recorded holograms $I_{1}$ and $I_{2}$, term (b) is the desired complex amplitude and term (c) expresses the influence of both the object and its opposite located at a distance $2 d$ upstream (figure 5, line (i) to (iv)). Equation 6 can not be analytically inversed as term (c) has many zeros in Fourier plane. A pseudo-inverse using an appropriate regularization can however by searched. Both modulus and phase of term (b) are obtained through deconvolution of equation 6 although only the phase is unknown. The error on the modulus can therefore be exploited as a parameter to adapt the inversion regularization.

\subsubsection{Physical approach: inverse filter inspired by Fresnel diffraction}

Term (a) (fig. 5, line (iv)) contains the searched complex amplitude that is created by the object located in $-z$. This complex amplitude however is polluted by a negative contribution of a virtual object located in $-z-2 d$. An approximation of this latter contribution can be computed through the propagation of term (a). The virtual object can then be pushed back to $-z-4 d:(a)+(a) * \underline{h_{2 d}}=(b) *\left(\delta-\underline{h_{4 d}}\right)$ (see figure 5). This operation can be reiterated in order to reject the virtual object to any position $-z-2 \overline{N d}$ :

$$
(a) * \sum_{n=0}^{N-1} \underline{h_{2 n d}}=(b) *\left(\delta-\underline{h_{2 N d}}\right) .
$$




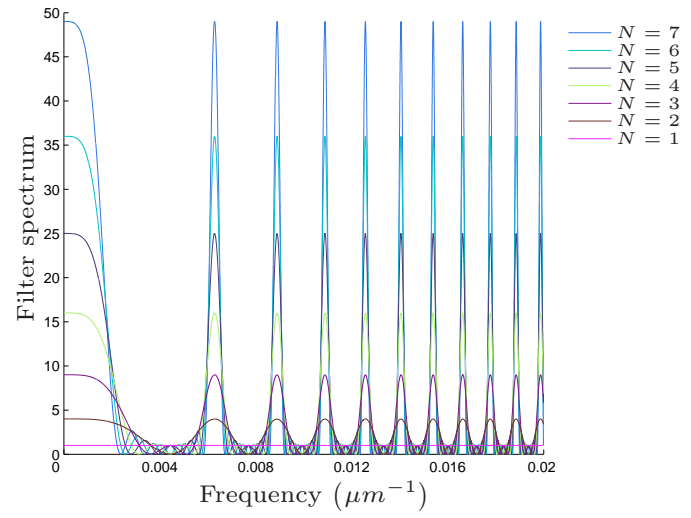

(a) Spectrum of the inverse filters

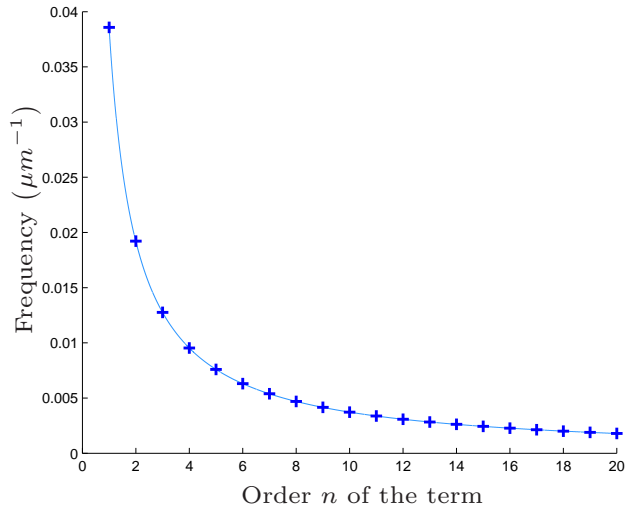

(b) Shanon sampling frequency for each term

Figure 6. Inverse filter characteristics: (a) Fourier spectrum for an increasing number $N$ of terms in the inverse filter expression; (b) Maximum frequency for a good sampling of the $n^{\text {th }}$ term of the inverse filter transfer function.

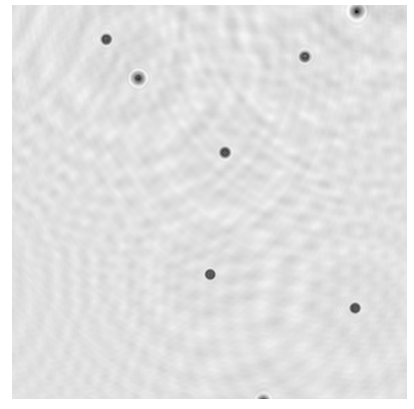

(a) Classical reconstruction $(z=150 \mathrm{~mm})$

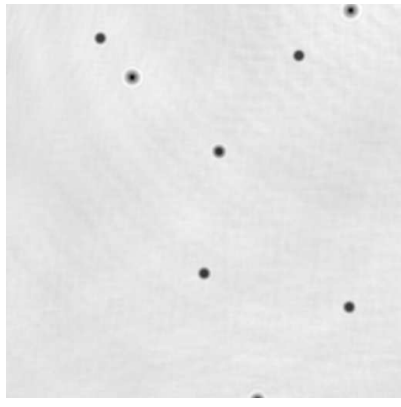

(b) Reconstruction after inverse filtering $(z=150 \mathrm{~mm})$

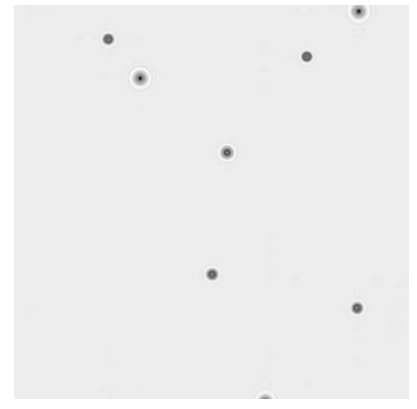

(c) Reconstruction using the known phase $(z=150 \mathrm{~mm})$

Figure 7. Twin-image reduction using inverse filtering: (a) without reduction; (b) using the described inverse filter $(N=22)$; (c) perfect reduction (simulation).

The amplitude of the wave an object diffracts is proportional to the inverse of the recording distance. The amplitude of the remaining term $(b) * \underline{h_{2 N d}}$ therefore lowers like $1 /(z+2 N d)$ does and only the first terms of the sum are required to find a good estimate for $(b)$.

\subsubsection{Implementation and illustration}

The Fourier transform of term (c) has many zeros. There are therefore stability issues for inversion of equation 6. The proposed inverse filter has a spectrum that evolves as illustrated in figure 6(a) when the number of terms $N$ increases. The spectrum tends to a ray spectrum whose singularities are responsible for the instability. Only a limited number of terms have to be used for inverse filtering to preserve the quality of the deconvolution. The determination of this number can be achieved in comparing the squared modulus of deconvolved term (b) and hologram $I_{1}$.

Each term appearing in the sum of equation 7 has to fullfil good sampling conditions. The higher the order of the term, the more it must be windowed to prevent from aliasing. Figure 6(b) illustrates the cutting frequency dependency on term order.

The inverse filtering procedure is applied on a synthetic particle hologram. A reconstructed plane is displayed on figure 7 (b) to compare with both classical (i.e. without twin-image reduction) reconstruction, figure 7(a), and perfect (computed from exact phase) reconstruction, figure $7(\mathrm{c})$. 


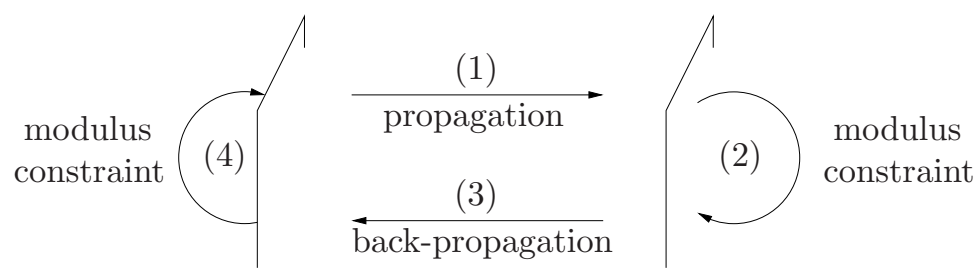

Figure 8. Gerchberg-Saxton iterative phase retrieval

\subsection{Iterative propagation approach: Gerchberg-Saxton derived method}

\subsubsection{Principle of the phase retrieval method}

The Gerchberg-Saxton iterative algorithm refers to a wide class of methods. ${ }^{21}$ It requires either to know two images (the modulus of the object and the modulus of its Fourier transform) or only one image (the modulus of the Fourier transform) plus a constrain (on the object). Although the version with a single image has been adapted to the case of inline holography of a real and plane object ${ }^{5}$, the most interesting version for digital holography is the two-images version ${ }^{6}$ as it applies to the more general class of $3 \mathrm{D}$ objects (and equivalently complex-valued transmittance objects).

Phase retrieval in the in-line holography context requires to adapt Gerchberg-Saxton's algorithm. The two recorded images correspond to two Fresnel transforms (at different scales) of the object. The correspondance from one plane to another is done via conjugate (complex) Fresnel Transforms. The principle of the iterative phase reconstruction is depicted in figure 8. Algorithm initialization is discussed in the following section. The algorithm consists in four steps ${ }^{7,21}$ : (1) propagate the estimate of complex amplitude in the first hologram plane to the second hologram plane; (2) replace the modulus of the resulting complex amplitude with the square root of the second hologram; (3) back-propagate this new estimate to the first hologram plane; and (4) replace the modulus of the computed complex amplitude with the square root of the first hologram. In equation, this is for the $k$ th iteration, using similar formalization as in Ref. 21:

$$
\begin{aligned}
\underline{A_{2_{k}}} & \equiv\left|\underline{A_{2_{k}}}\right| \exp \left(j \varphi_{2}\right)=\underline{A_{1_{k}}^{\prime}} * \underline{h_{d}}, \\
\frac{A_{2_{k}}^{\prime}}{\overline{A_{1_{k}}}} & \equiv\left|\underline{\sqrt{I_{2}}}\right| \exp \left(j \varphi_{2}\right), \\
\underline{A_{1_{k}}} \mid \exp \left(j \varphi_{1}\right)=\underline{A_{2_{k}}^{\prime}} * \underline{h_{-d}}, & =\mid \underline{\sqrt{I_{1}}} \exp \left(j \varphi_{1}\right),
\end{aligned}
$$

where $I_{1}$ (resp. $I_{2}$ ) is the intensity of the first (resp. second) hologram, $d$ is the distance between the holograms, $A_{1_{k}}^{\prime}$ and $\varphi_{1}$ (resp. $A_{2_{k}}^{\prime}$ and $\varphi_{2}$ ) are estimates of the complex amplitude and phase in the first (resp. second) hologram plane.

\subsubsection{Implementation and illustration}

In order to reach a good estimate of the phase in a small number of iterations, the first step has to give a reasonably good phase. The choice of the hologram to start the iterations with is therefore important. To the best of our knowledge, it has not been discussed previously (see Ref. 7). Let $I_{1}$ and $I_{2}$ be the two recorded holograms (resp. at distances $z_{1}$ and $z_{2}$ ). According to equation 5 , the propagation of centered hologram $I_{1}$ can be expressed as:

$$
\tilde{I}_{1} * \underline{h_{z_{2}-z_{1}}} \approx-\underline{a} * \underline{h_{z_{2}}}-\underline{a}^{*} * \underline{h_{z_{2}-2 z_{1}}} .
$$

Let's write the ratio of the amplitude of the first term (induced by the real object) to the amplitude of the second term (corresponding to the propagation of the virtual object):

$$
\frac{-z_{2}+2 z_{1}}{z_{2}}=2 \frac{z_{1}}{z_{2}}-1
$$




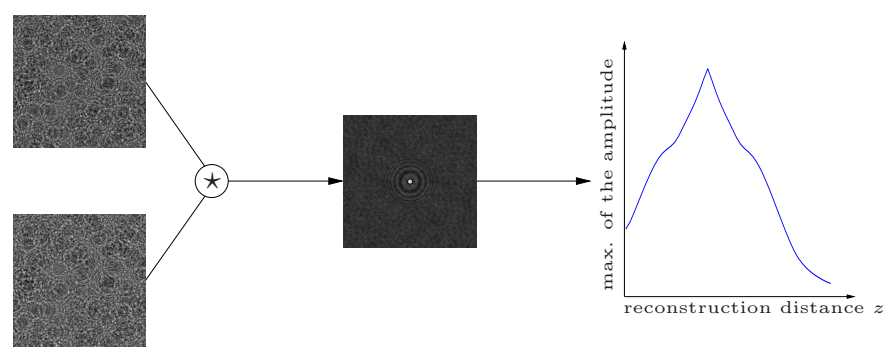

Figure 9. Inter-hologram distance retrieval method based on cross-correlation

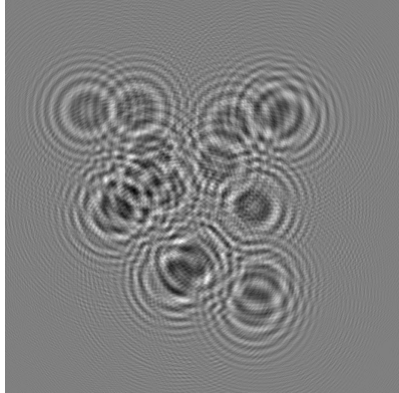

(a)

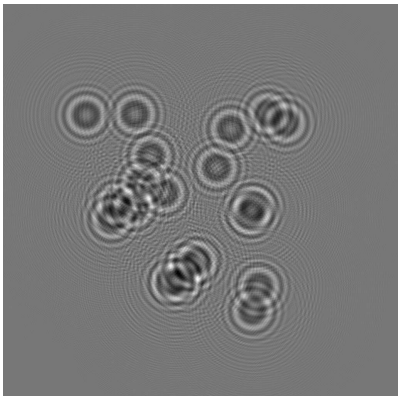

(b)

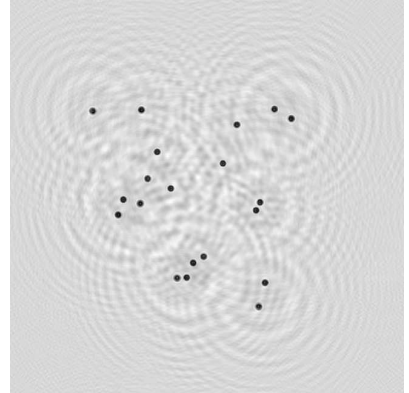

(c)

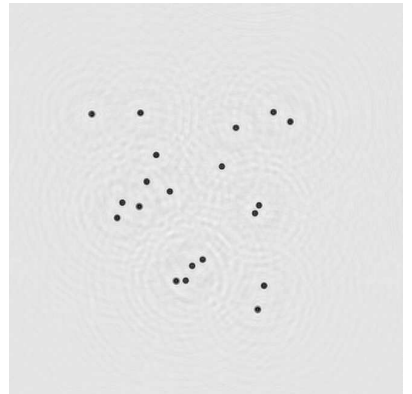

(d)

Figure 10. Twin-image reduction using Gerchberg-Saxton algorithm

As we aim at reaching the phase of the first term, we have to set $z_{1}$ and $z_{2}$ so as to maximize the aforementionned ratio. Therefore, $z_{1}$ has to be larger than $z_{2}$. The hologram to start the iterations with should be the farther from the object. The distance between the holograms should be chosen as large as possible.

This distance has also to be chosen accordingly to both the sensor and pixel sizes. On the one hand, if the holograms are recorded too close one another, their difference will not be significant and equation 9 ratio will stay close to one. On the other hand, the higher the inter-hologram distance, the worse the reconstructed phase at the image borders.

Another issue is the precision at which this distance is known. Let's note here that it can be easily retrieved using the following procedure, illustrated in figure 9: (1) substract its mean to each hologram; (2) cross-correlate the first hologram with the second; (3) find the distance $d_{\max }$ which maximizes $\underline{h_{d}} *\left(\tilde{I}_{1} \star \tilde{I}_{2}\right)$.

The sub-pixel lateral position of the maximum can also be used to correct misalignment of the hologram (i.e. hologram registration).

Care has also to be taken to fulfill a correct numerical propagation without introducing periodization artifacts. Both adapted anti-aliasing and zero padding must be applied.

Figure 10 shows the classical reconstruction (fig. 10(c)) obtained from hologram (b). Gerchberg-Saxton algorithm applied on holograms (a) and (b) simulated respectively at $190 \mathrm{~mm}$ and $100 \mathrm{~mm}$ provides the twinimage attenuated image $(\mathrm{d})$. 


\section{TWIN-IMAGE CLEANING BY ITERATIVE NON-LINEAR FILTERING OF A SINGLE HOLOGRAM}

The need of more than one hologram is a constraint which can induce a more complex system and some additional treatments, especially for three dimensional objects in motion and deformation. Such a situation can be encountered in Digital Holography Particle Image Velocimetry which is an important application of in-line holography. Another twin-image reduction approach involving a single hologram therefore has to be carried out.

\subsection{Principle}

The twin-image can not be reduced for 3D objects using a linear filtering approach on a single hologram (see section 3.1). In the case of small objects with strong transmittance contrast, a spatial separation scheme between the in-focus and the out-of-focus images can be attempted. Iterative algorithm have been proposed to suppress the twin-image in the case of plane objects (see Ref. 8), we describe a relatively different approach here.

Figure 3(b) shows a reconstructed plane of a numerically simultated hologram of three objects. Two of the objects lie in the same plane, the other one is located $20 \mathrm{~mm}$ above. In the reconstruction plane, two objects are in focus and the third one is slightly out of focus (see section 2.3). Although the first two objects are easy to separate from their twin rings, the third one can not be easilly extracted. Volume objects therefore require 3D processing to separate the twin-images.

In our process to suppress twin-image noise, the signal consists in the in-focus real images while the noise is formed by out-of-focus twin-images. However it should be noted that the in-focus and out-of-focus images contain the same information and are transformed one into another when propagating towards the symmetrical plane with respect to the hologram. Moreover, we know a priori that all the objects lie the same side of the sensor. The out-of-focus rings therefore can be suppressed by masking their focalized counterpart in the virtual image side.

Let us note that the following two transformations are equivalent:

(a) (1) reconstruct plane $z_{r}$; (2) delete focalized objects; (3) back propagate towards the hologram;

(b) (1) reconstruct plane $z_{r}$; (2) delete all but focalized objects; (3) back propagate towards the hologram; (4) substract the complex amplitude computed in step (3) to the hologram.

Transformation (b) however is more interesting as it does not involve the out-of-focus image that could overflow the reconstructed image borders.

The general principle therefore is to clean the twin-image rings by substracting a corrective complex amplitude to the hologram. This complex amplitude is computed through backpropagation of in-focus virtual objects. These virtual objects are selected on a thresholding basis. The corrections are applied iteratively to the hologram to ensure efficient twin-image cleaning.

As we underlined at the begining of this section, three dimensional objects require an appropriate processing. The masking operation can not be applied on a single plane. The principle we use to extend the algorithm to three dimensional object is inspired from non-linear wavelets filtering. Denoising is performed with wavelets by applying a threshold on the transformed images at different scales and then by summing up all the reversetransformed images. Transposed into our problem, the denoising operation consists in summing up all the propagated planes after thresholding the virtual-side volume.

\subsection{Algorithm description}

The algorithm consists in two major steps: 
A- Mask construction: In this step a three dimensionnal mask is created. A limited number $N$ of planes is chosen and those planes are reconstructed. They are thresholded at a high enough level to prevent from too many false detections. The binary mask then is dilated and low pass filtered in order to reduce border effects during step B:

- reconstruct $N$ planes (real side of the hologram),

- threshold the volume (set the mask to 1 inside the objects and to 0 elsewhere),

- dilate slightly by a 2 pixels radius disk,

- filter the mask.

B- In-mask cleaning: The aim of this step is to clean the out-of-focus image. To do that we suppress the influence of the virtual particles. They are selected using the mask on a plane by plane basis:

For each plane, do

- reconstruct the plane (virtual side of the hologram),

- multiply the complex amplitude and the mask,

- back-propagate this amplitude in the hologram plane,

- substract computed amplitude to the hologram.

Then, move on to the next plane.

Two options are possible to reiterate this algorithm. Either the two steps are applied with a decreasing threshold level, as the signal to noise ratio is increasing (step A, second point); or only step B is reiterated. The modulus constraint of the recorded hologram can be reintroduced after each B step, however, thanks to the fast convergence of the algorithm, this is not necessary in practice.

Figure 11 illustrates the algorithm scheme. The twin-image cleaning process appears more clearly: images at the right side contain the real objects while images at the left side contain out-of-focus images of the real objects and in-focus remainder of the virtual objects. The twin-image therefore is reduced using a non linear filtering in the twin (i.e. virtual) half-space. The number of analysed planes can be limited as the cleaning operation can act efficiently even in a slightly out-of-focus plane. Figure 12 displays the evolution of both in-mask (i.e. cleaning plane) and in-focus amplitudes (only the real part is shown) during the cleaning of a virtual particle. The analysed plane is $2 \mathrm{~mm}$ beyond the in-focus plane (in practice, the in-focus plane location would be unknown). After 5 iterations, the virtual object appears to be efficiently deleted (figure 12, first row of images, last column). The in-mask amplitude tends to zero (figure 12, last row of images, last column). The energy of the correction applied on the hologram at each iteration decreases as depicted in the semi-logarithmic plot of figure 13.

\subsection{Results}

Let us illustrate results we can get with this algorithm with three examples.

Synthetic hologram of a simple three dimensional distribution of small objects The object studied here is made of three planes located at $20 \mathrm{~mm}$ distance each. The simulated hologram is recorded at $100 \mathrm{~mm}$ from the closest plane. Figure 14(a) gives a diagram representing the object. The corresponding computed hologram is displayed in figure 14(b). Figures 14(c) to (e) show the three reconstructed planes using the classical reconstruction algorithm (see section 1 ). The reconstructed planes after twin-image cleaning are given in figure 14(f) to (h). The twin-image reduction appears to be very efficient. 


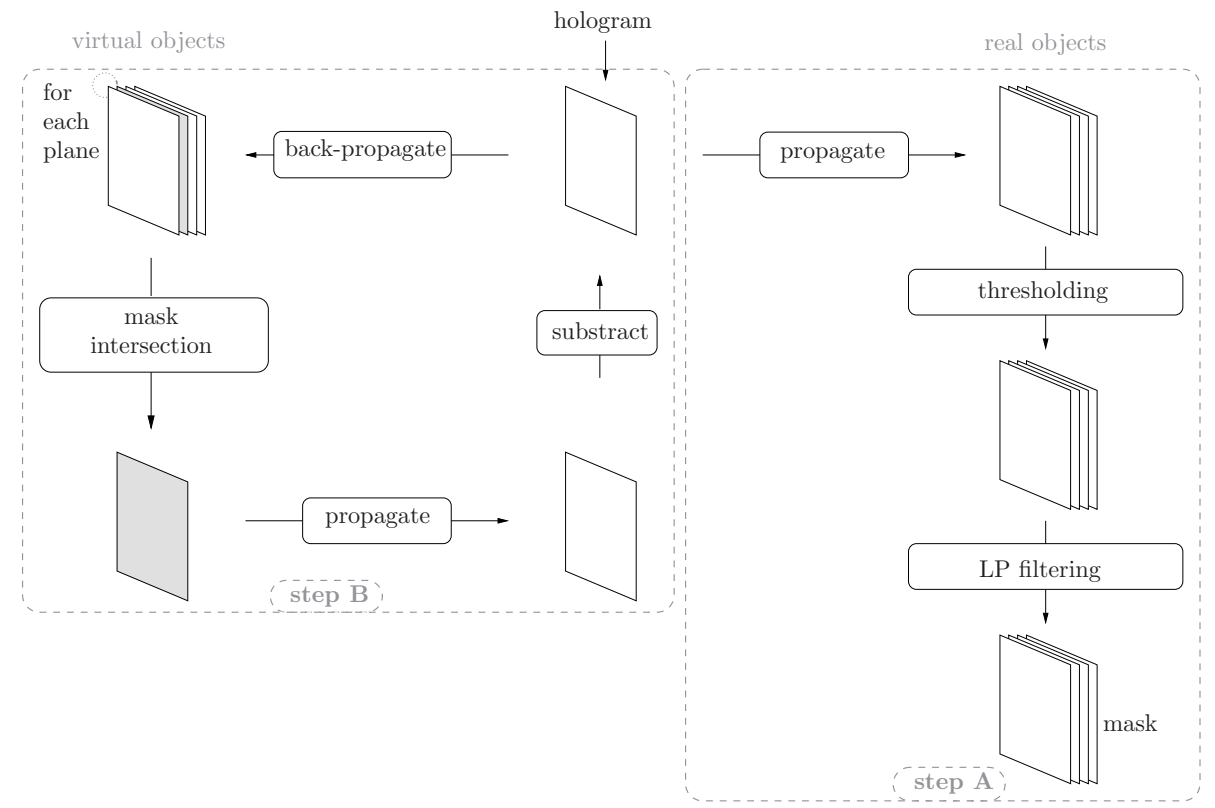

Figure 11. Twin-image cleaning scheme

iteration step

1

2

3

4

5

in-focus plane

(virtual objects side)

(zoomed in)
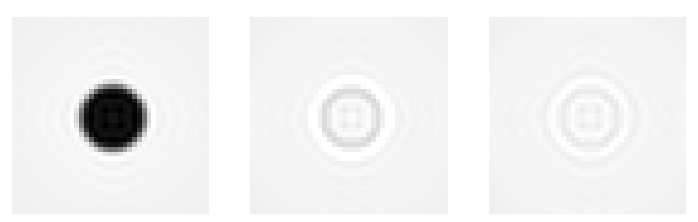

cleaning plane

(virtual objects side)

(zoomed in)
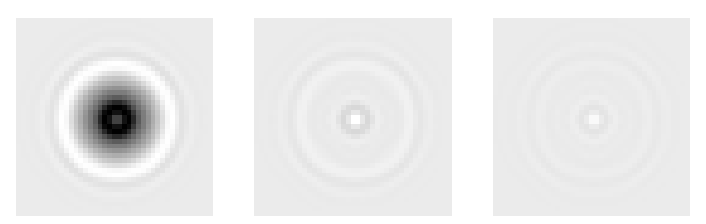

Figure 12. Cleaning process evolution through the first iterations

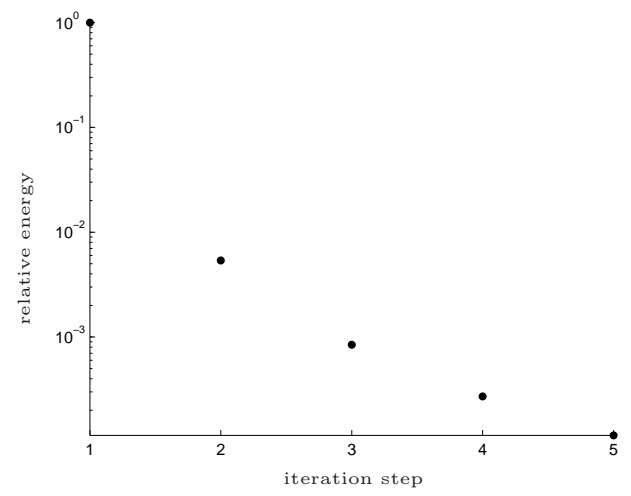

Figure 13. Energy of the corrections at each iteration step (semi-logarithmic plot) 


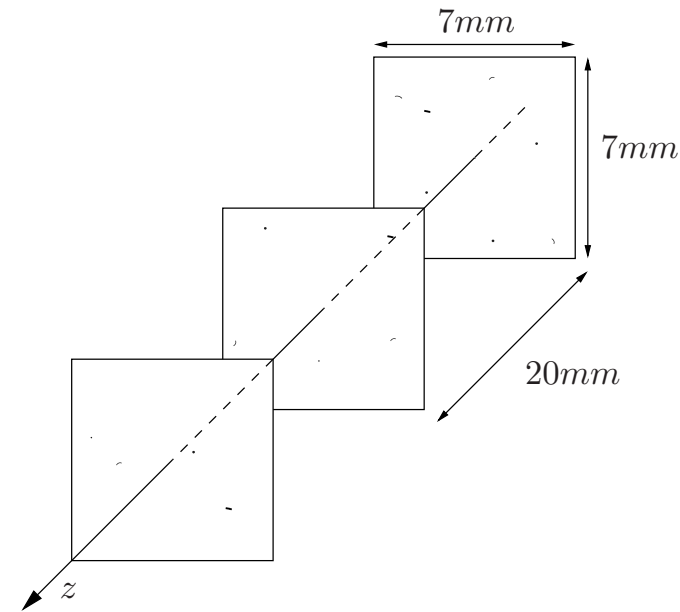

(a)

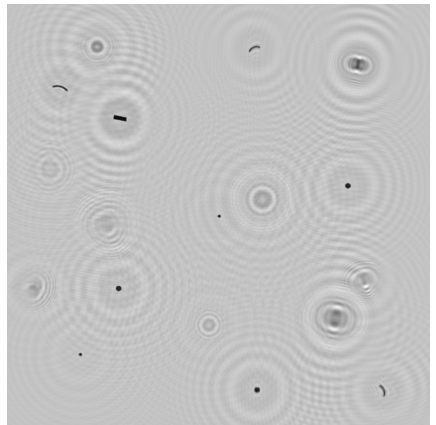

(c)

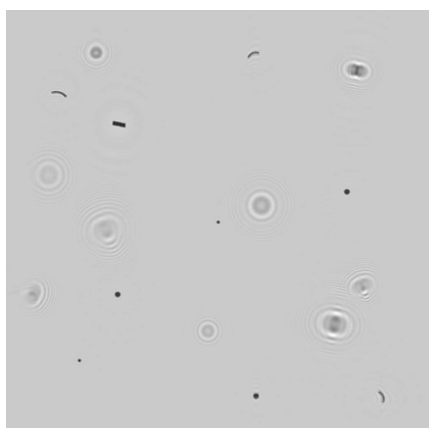

(f)

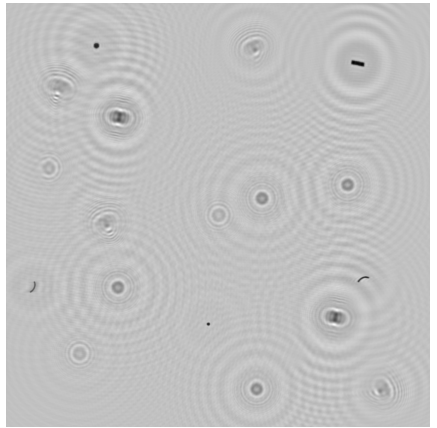

(d)

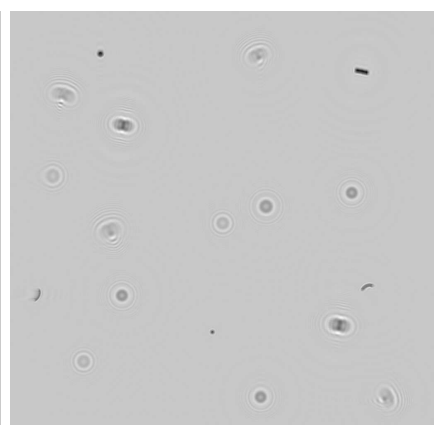

(g)

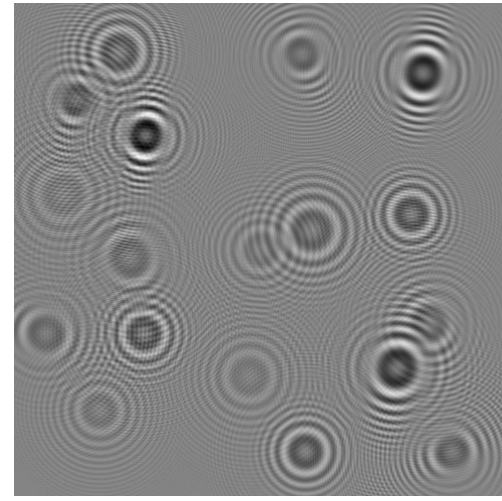

(b)

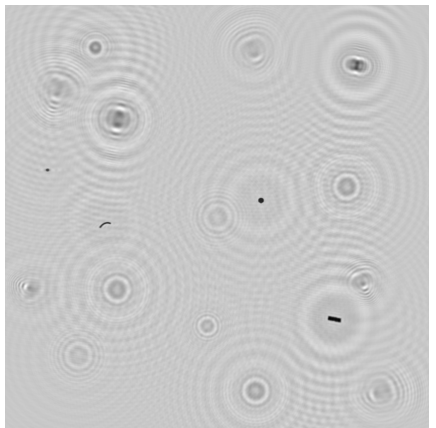

(e)

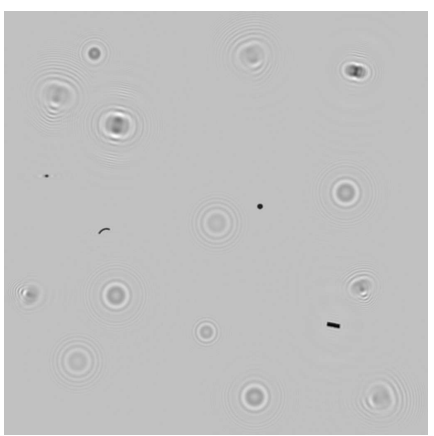

(h)

Figure 14. Twin-image cleaning applied on a simple synthetic hologram 


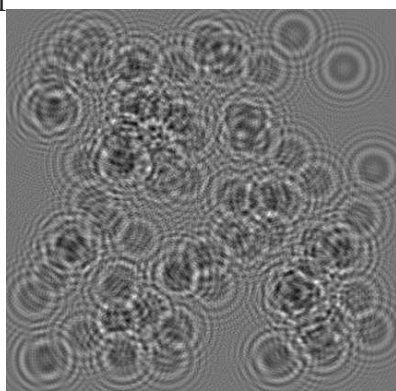

(a)

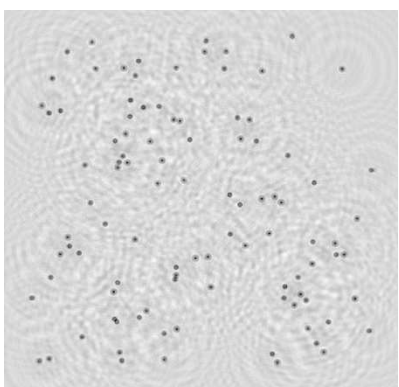

(b)

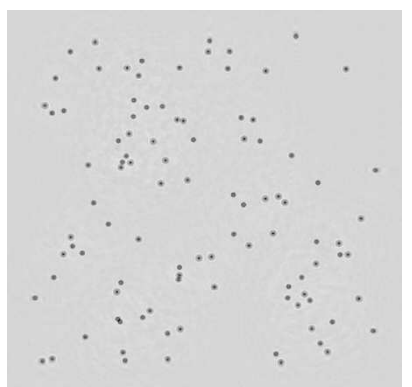

(c)

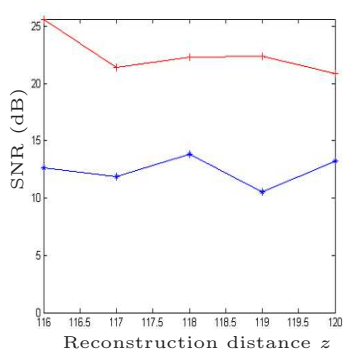

(d)

Figure 15. Twin-image cleaning applied on a synthetic hologram of 100 particles

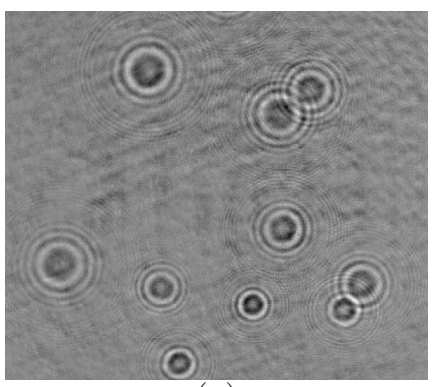

(a)

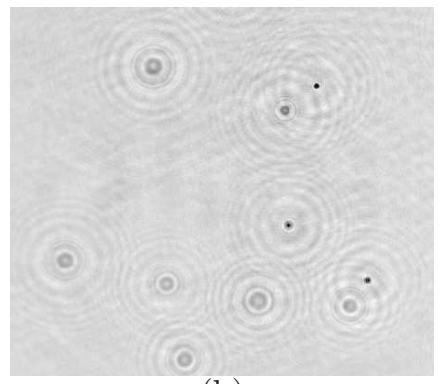

(b)

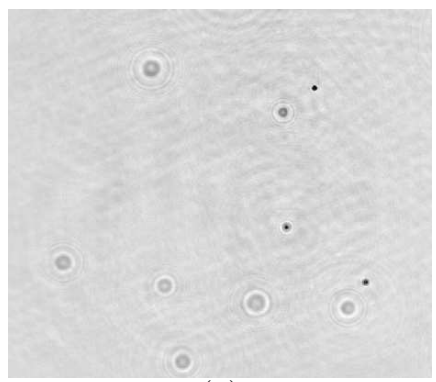

(c)

Figure 16. Twin-image cleaning applied on an experimental hologram of a water spray

Simulated hologram of opaque particles One hundred opaque spheres (diameter $90 \mu \mathrm{m}$ ) were spread in a $6 \times 6 \times 6 \times \mathrm{mm}^{3}$ volume. An hologram was numerically generated at a distance $z=120 \mathrm{~mm}$. Figure 15 (a) displays the hologram. Classically reconstructed and twin-image cleaned planes are displayed in figures 15(b) and 15(c) respectively. Five "cleaning" planes were used in the algorithm. Figure 15(d) gives the signal to noise ratio in each 5 plane before (lowest curve) and after (highest curve) twin-image reduction. The twin-image reduction algorithm has increased the SNR by $10 \mathrm{~dB}$.

Experimental hologram of a water spray A cloud of water droplets was produced by a water spray. A hologram was recorded on a camera $(1280 \times 1024$ pixels $)$ using a YAG pulsed laser $($ lambda $=532 n m$, pulse duration $=7 n s)$. The dropplets were located within a bounding volume of $60 \mathrm{~mm}$ depth. Their sizes is roughly $90 \mu \mathrm{m}$. Figure 16(a) shows the experimental hologram. The classical reconstruction is given in figure 16(b). The reconstruction after twin-image reduction is displayed in figure 16(c). The twin-image has been strongly attenuated.

\section{DISCUSSION}

Whatever the method chosen, let us notice that as long as the twin-image is strongly reduced, the non-linear term in the expression of the hologram intensity (see equation (4)) can be suppressed as described in Ref. 3.

In the given description of the three possible methods for twin-image reduction, we paid specific attention to adapt the algorithms to 3D objects. Improvements were proposed to the classical approaches. Each method has been reformulated as a phase retrieval problem in the hologram plane. This formulation gives control on the stability of the inverse filter in the linear filtering approach. It also permits a natural extension to the case of $3 \mathrm{D}$ objects. 
Gerchberg-Saxton's iterative approach has been reviewed and the problem of the choice of the initial value has been raised. We have shown that the farthest hologram should be used to get the best phase estimate after the first step of the algorithm.

An iterative algorithm requiring a single hologram has been introduced. The results given on both synthetic and experimental holograms are promising. We are now working on improvements of this technique to address the following challenge: process higher and higher concentrated holograms!

\section{REFERENCES}

1. Y. Zhang and X. Zhang, "Reconstruction of a complex object from two in-line holograms," Optics Express, vol. 11, Issue 6, p.572 11, pp. 572-+, June 2003.

2. L. Onural and P. Scott, "Digital decoding of in-line holograms," Optical Engineering 26(11), pp. 1124-1132, 1987.

3. K. A. Nugent, "Twin-image elimination in Gabor holography," Optics Communications 78, pp. 293-299, Sept. 1990.

4. M. H. Maleki and A. J. Devaney, "Noniterative reconstruction of complex-valued objects from two intensity measurements," Optical Engineering 33(10), pp. 3243-3253, 1994.

5. G. Liu and P. Scott, "Phase retrieval and twin-image elimination for in-line fresnel holograms," JOSA $A$ 4(1), pp. 159-165, 1987.

6. S. Grilli, P. Ferraro, S. D. Nicola, A. Finizio, G. Pierattini, and R. Meucci, "Whole optical wavefields reconstruction by digital holography," Optics Express 9(6), pp. 294-302, 2001.

7. Y. Zhang, G. Pedrini, W. Osten, and H. J. Tiziani, "Whole optical wave field reconstruction from double or multi in-line holograms by phase retrieval algorithm," Optics Express, vol. 11, Issue 24, p.3234 11, pp. 3234-+, Nov. 2003.

8. G. Koren, F. Polack, and D. Joyeux, "Iterative algorithms for twin-image elimination in in-line holography using finite-support constraints," Optical Society of America Journal A 10, pp. 423-433, Mar. 1993.

9. J. Goodman, Introduction to Fourier Optics, Mc Graw-Hill, 1996.

10. T. Kreis, M. Adams, and W. Juptner, "Methods of digital holography : A comparison," in SPIE97, 3098, pp. 224-233, (Munich, Germany), 1997.

11. M. Liebling, T. Blu, and M. Unser, "Fresnelets : New multiresolution wavelet bases for digital holography," IEEE Transactions on image processing 12(1), pp. 29-43, 2003.

12. L. Onural and M. Kocatepe, "Family of scaling chirp functions, diffraction, and holography," IEEE Transactions on Signal Processing 43(7), pp. 1568-1578, 1995.

13. C. Buraga-Lefebvre, S. Coëtmellec, D. Lebrun, and C. Özkul, "Application of wavelet transform to hologram analysis: three-dimensional location of particles," Optics and Lasers in Engineering 33(6), pp. 409-421, 2000.

14. Kreis, Handbook of Holographic Interferometry, Wiley-VCH, Berlin, 2004.

15. M. Unser, A. Aldroubi, and M. Eden, "On the asymptotic convergence of b-spline wavelets to gabor functions," IEEE Transactions on Information Theory 38(2), pp. 864-872, 1992.

16. G. Pan and H. Meng, "Digital holography of particle fields: reconstruction by use of complex amplitude," Applied Optics 42, pp. 827-833, 2003.

17. Leith and Upatnieks, "Reconstructed wavefronts and communication theory," JOSA A 52(10), pp. 11231130, 1962.

18. I. Yamaguchi and T. Zhang, "Phase-shifting digital holography," Optics Letters 22(16), 1997.

19. S. Murata and N. Yasuda, "Potential of digital holography in particle measurement," Optics and Laser Technology 32(7 8), pp. 567-574, 2000.

20. H. Meng, W. Anderson, F. Hussain, and D. Liu, "Intrinsic speckle noise in in-line particle holography," JOSA A 10(9), pp. 2046-2058, 1993.

21. J. Fienup, "Phase retrieval algorithms : a comparison," Applied Optics 21(15), pp. 2758-2769, 1982. 


\section{Copyright notice}

This paper was published in the Proceedings of SPIE and is made available as an electronic reprint with the permission of SPIE. The paper can be found at the following URL on the SPIE website :

http://spiedl.aip.org.

The full reference of the article is :

"Twin-image noise reduction by phase retrieval in in-line digital holography," L. Denis, C. Fournier, T. Fournel, and C. Ducottet, presented at Wavelets XI, SPIE's Symposium on Optical Science and Technology, San Diego, CA, USA, 2005.

(doi : 10.1117/12.617405). 